\title{
Awake craniotomy - the anaesthesiologist's view
}

\begin{abstract}
Inga Mladić Batinica ${ }^{1}$, K. Rotim² ${ }^{2}$ T. Sajko ${ }^{2}$, M. Zmajević Schonwald ${ }^{2}$, S. Salkičević ${ }^{3}$
${ }^{1}$ Department of Anaesthesiology, Intensive Care and Pain Therapy, University Hospital Center "Sisters of Mercy", Zagreb, Croatia; ${ }^{2}$ Department of Neurosurgery, University Hospital Center "Sisters of Mercy", Zagreb, Croatia; ${ }^{3}$ Department of Psychology, Faculty of Humanities and Social Sciences, University of Zagreb, Croatia

E-mail: ingamblue@gmail.com
\end{abstract}

Background: Compared with surgery under general anaesthesia (GA), awake craniotomy (AC) is associated with advantageous outcomes. For anaesthesiologist it is a challenge because there is no standardized anaesthestic technique.

Clinical case-series: During last three years, AC with monitored anaesthesia care (MAC) is successfully implemented in Croatia. We present single centre experience with thirteen patients (age 32-64 yrs; 7M, 6F; ASA II).

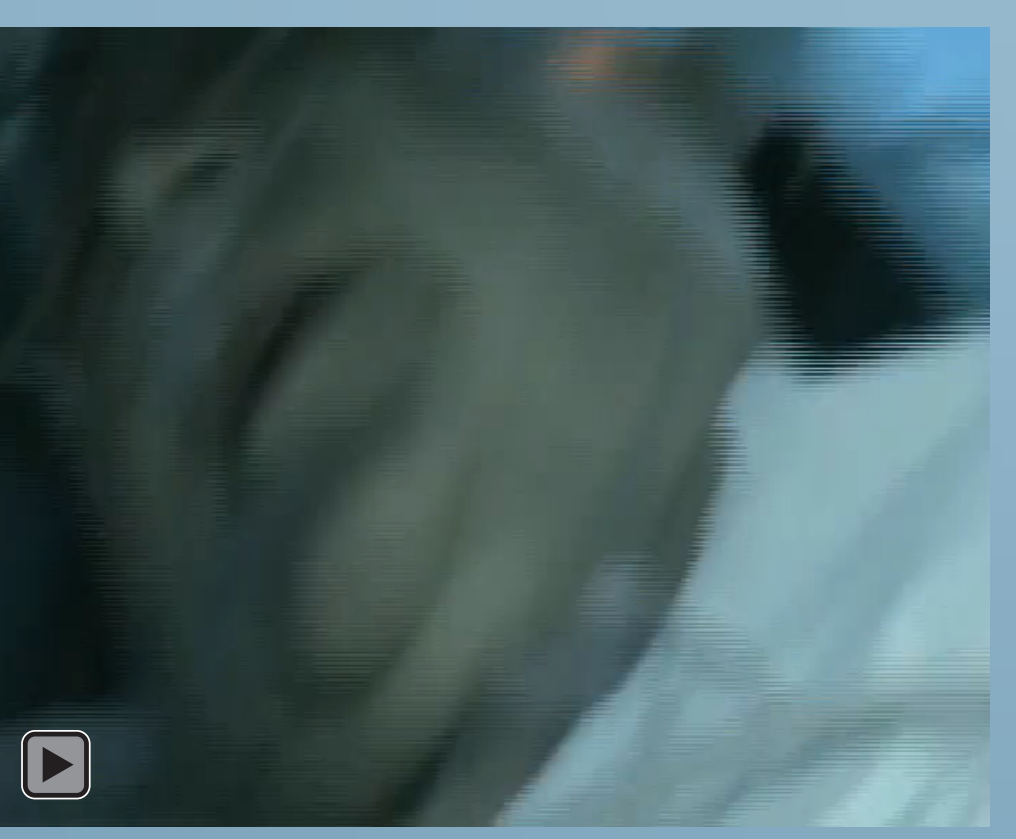

Discussion: Implementing AC is challenging for anaesthesiologist. It is difficult to differentiate the benefits related to anaesthetic care from those attributable to surgical technique.
Patients were sedated and breathed spontaneously. We used TCl pumps for titration of remifentanil and propofol, and mixture of $0.5 \%$ bupivacaine and $2 \%$ lidocaine with adrenalin for local infiltration.

Monitoring included: ECG, IBP and NIBP, SPO2, RR, ETCO2, BIS and urinary output. Maximum alert and minimally stressed patients were optimal for awake brain mapping.
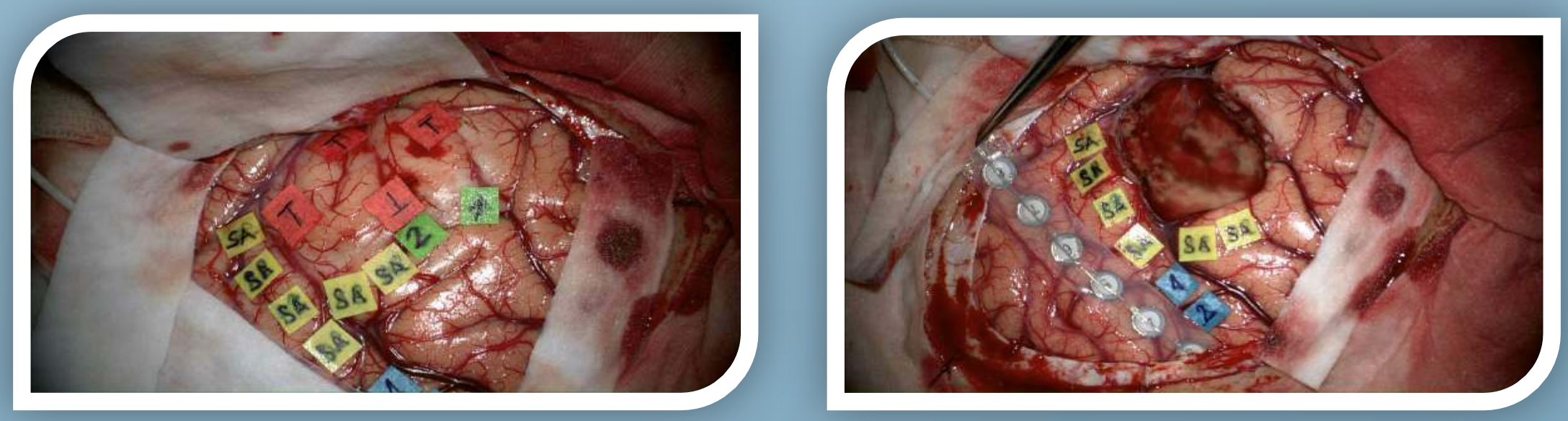

Learning points: Crucial steps in establishment of an AC is creation of a competent neuroanaesthesiologist. Drugs fine titration, accurate respiratory function monitoring, good plan in dealing with respiratory complication and constant training in management of difficult airways are milestones of favourable outcome. In view of that, anaesthesiologist contribution to AC is essential.

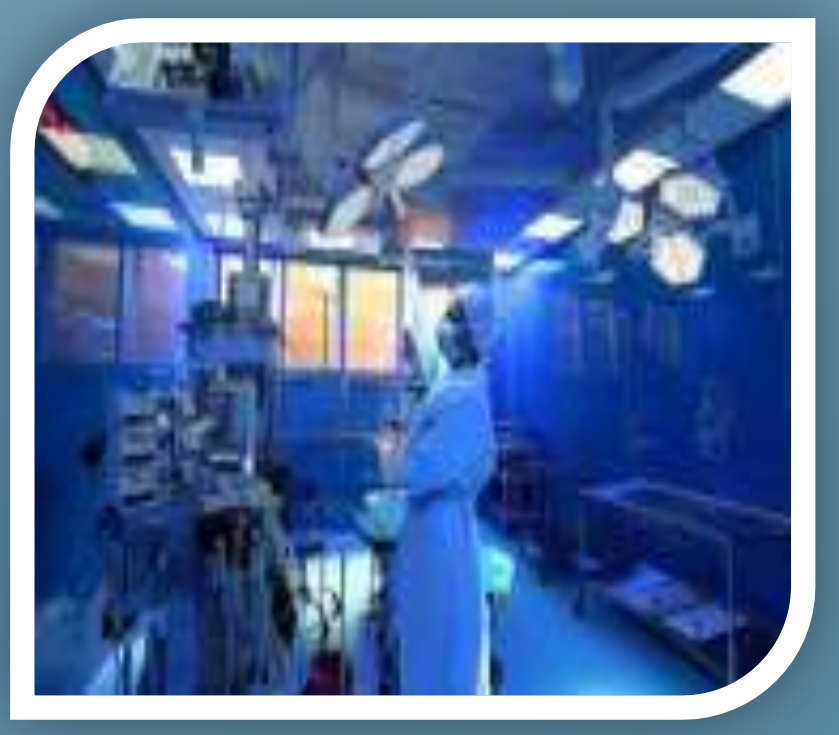

